\title{
Sorafenib dose escalation in treatment-naive patients with metastatic renal cell carcinoma: a non-randomised, open-label, Phase 2b study
}

\author{
Martin E. Gore*, Robert J. Jones ${ }^{\dagger}$, Alain Ravaud", Markus Kuczyk ${ }^{\S}$, Tomasz Demkow", \\ Alessandra Bearz**, JoAnn Shapiro ${ }^{\dagger \dagger}$, Uwe Phillip Strauss ${ }^{\sharp}$ and Camillo Porta ${ }^{\S \S}$
}

*Royal Marsden Hospital, London, 'Beatson West of Scotland Cancer Centre, University of Glasgow, Glasgow, UK, * Hôpital Saint-André CHU, Bordeaux, France, 's Medizinische Hochschule Hannover, Hannover, "Bayer Vital GmbH, Leverkusen, Germany, "Centrum Onkologii-Instytut im. Marii Sklodowskiej-Curie, Warszawa, Poland, **IRCCS Centro Rif Oncologico, Aviano, ${ }^{\xi}$ IRCCS Policlinico San Matteo, Medicina Interna ed Oncologia Medica, Pavia, Italy, and "H'Bayer HealthCare Pharmaceuticals Inc., Whippany, NJ, USA

Trial Registration: ClinicalTrials.gov identifier NCT00618982, www.clinicaltrials.gov. This article was presented in part as an oral presentation at the American Society of Clinical Oncology Annual Meeting, 3-7 June 2011, Chicago, IL, USA (abstract 4609).

\section{Objective}

To assess the efficacy and safety of sorafenib dose escalation in metastatic renal cell carcinoma (mRCC).

\section{Patients and Methods}

Intra-patient dose escalation may enhance the clinical benefit of targeted anticancer agents in metastatic disease. In this non-randomised, open-label, Phase $2 \mathrm{~b}$ study, treatment-naive patients with mRCC were initially treated with the standard oral sorafenib dose [400 mg twice daily (BID)]. Two dose escalations were planned, each $200 \mathrm{mg}$ BID after 28 days at the prior level. Dose reductions, interruptions, or delayed escalations were used to manage adverse events (AEs). The primary endpoint was objective response rate (ORR) in the modified intent-to-treat (mITT) population, which comprised patients with $\geq 6$ months of treatment including $\geq 4$ months of therapy at their highest tolerated dose. Secondary endpoints included progression-free survival (PFS) and safety.

\section{Results}

In all, 83 patients received sorafenib. The dose received for the longest duration was 400,600, and $800 \mathrm{mg}$ BID in 48.2\%,
$15.7 \%$, and $24.1 \%$ of patients, respectively. The ORR was $44.4 \%[n=8 / 18$; 95\% confidence interval (CI) 21.5-69.2] and $17.9 \%(n=12 / 67 ; 95 \%$ CI 9.6-29.2) in the mITT and ITT populations, respectively. The median (95\% CI) PFS was 7.4 (6.0-11.7) months (ITT). The most common AEs of any grade were hand-foot skin reaction $(66.3 \%)$ and diarrhoea (63.9\%).

\section{Conclusion}

Sorafenib demonstrated clinical benefit in treatment-naïve patients with mRCC. However, relatively few patients could sustain doses of $>400 \mathrm{mg}$ BID. There was evidence that, where tolerated, escalation from the standard sorafenib dose may have enhanced clinical benefit. However, this study does not support dose escalation for most patients with treatmentnaïve mRCC. Alternative protocols for sorafenib dose escalation could be explored.

\section{Keywords}

dose escalation, renal cell carcinoma, sorafenib, targeted therapy, tyrosine kinase inhibitor, \#KidneyCancer

\section{Introduction}

The advent of molecularly targeted agents brought welcome advances in the treatment of metastatic RCC (mRCC).

However, more effective approaches to this ultimately incurable disease are needed. One strategy is intra-patient dose escalation of agents that have demonstrated efficacy and tolerability.
Sorafenib, an oral inhibitor of several kinases involved in tumour angiogenesis and cell proliferation, is approved in differentiated thyroid carcinoma, hepatocellular carcinoma, and advanced/mRCC [1-6]. The pivotal TARGET trial in patients pre-treated with cytokine therapy demonstrated efficacy of sorafenib $400 \mathrm{mg}$ twice daily (BID); this subsequently became the approved regimen [3-6]. 
Attempts to enhance clinical outcome investigated sorafenib doses $>400 \mathrm{mg}$ BID [7-9]. A Phase 2 study in mRCC in which $\sim 50 \%$ of patients had received prior systemic therapy but no tyrosine kinase inhibitor escalated the sorafenib dose at 28-day intervals to $600 \mathrm{mg}$ BID [in $92.9 \%$ of patients $(n=39 / 42)$ ], then $800 \mathrm{mg}$ BID [in $73.8 \%$ of patients $(n=31 / 42)$ ] [9]. The objective response rate (ORR) was $47.7 \%(n=21 / 44)$ and median progression-free survival (PFS) was 8.4 months [9]. These outcomes compared favourably with those of TARGET.

Further investigation of sorafenib dose escalation was therefore warranted. In the present study, we report the efficacy, safety, and tolerability from an open-label, Phase $2 b$ study of planned sorafenib dose escalation in treatment-naive patients with mRCC.

\section{Patients and Methods \\ Study Design and Patients}

This non-randomised, open-label, uncontrolled, international, multicentre, Phase $2 \mathrm{~b}$ study (ClinicalTrials.gov NCT00618982) recruited patients aged $\geq 18$ years with: histologically/cytologically confirmed metastatic clear cell RCC; no prior systemic therapy for RCC; Eastern Cooperative Oncology Group performance status (ECOG PS) 0 or 1; intermediate or good prognosis according to the Memorial Sloan-Kettering Cancer Center scale [10]; $\geq 1$ measurable lesion by CT or MRI according to Response Evaluation Criteria in Solid Tumors (RECIST) v1.0; life expectancy of $\geq 12$ weeks; prior nephrectomy; and adequate bone marrow, liver, and renal function assessed within 7 days prior to study treatment. Prior palliative radiotherapy to non-targeted metastatic lesions according to RECIST was permitted.

Exclusion criteria included: history of cardiac disease (congestive heart failure $>\mathrm{New}$ York Heart Association class 2 ); acute coronary disease (myocardial infarction $>6$ months before study entry was allowed); cardiac arrhythmias requiring anti-arrhythmic therapy ( $\beta$-blockers or digoxin were permitted); or uncontrolled hypertension; history of HIV infection or chronic hepatitis B or C; active clinically serious infections $>$ Grade 2; symptomatic metastatic brain or meningeal tumours; seizure disorders requiring medication; history of organ allograft; evidence or history of bleeding diathesis; deep vein thrombosis and/or pulmonary embolus within 12 months of treatment initiation; delayed healing of wounds, ulcers, or bone fractures; pre-existing thyroid abnormality; undergoing renal dialysis; previous or concurrent cancer distinct in primary site or histology from mRCC (except cervical carcinoma in situ, treated basal cell carcinoma, non-muscle-invasive bladder cancer, or any cancer curatively treated $>3$ years prior to study entry); pregnancy/ breastfeeding; inability to swallow oral medications; any prior systemic anticancer therapy; major surgery within 4 weeks prior to study entry; radiotherapy within 3 weeks of study drug initiation; biological response modifiers, e.g. granulocyte colony stimulating factor (G-CSF), within 3 weeks prior to study entry; or autologous bone marrow transplant or stem cell rescue within 4 months of study entry.

All patients provided written informed consent, and study approval was obtained from ethics committees (Table S1). The study was conducted in accordance with the World Medical Association Declaration of Helsinki, the International Conference on Harmonization guideline E6 for Good Clinical Practice, and local ethical and legal requirements.

\section{Treatment}

The initial dose was oral sorafenib $400 \mathrm{mg}$ BID. Two dose escalations were planned: to $600 \mathrm{mg}$ BID after 28 days at the starting dose, then to $800 \mathrm{mg}$ BID after another 28 days. The occurrence of any symptomatic adverse event (AE) $\geq$ Grade 3 (except nausea or vomiting) prevented dose escalation until the event resolved to Grade 1. Treatment continued until disease progression, unacceptable toxicity, withdrawal of consent, investigator's decision, or study end. Dose delays or reductions to 400 or $200 \mathrm{mg}$ daily were allowed to manage AEs (200 mg daily dose given as $400 \mathrm{mg}$ every other day or $200 \mathrm{mg}$ once daily). Dose modification or delay due to hypertension or dermatological, haematological, and non-haematological AEs was permitted according to specific criteria (Tables S2-S6).

Concomitant therapies were allowed: palliative radiotherapy to $\leq 10 \%$ of the patient's bone marrow provided that a target lesion was not irradiated and there was no progressive disease; G-CSF and other haemopoietic growth factors to manage acute toxicity, and secondary (not primary) prophylaxis with erythropoietin, providing these did not replace a required sorafenib dose reduction; other palliative/ supportive care, including bisphosphonates.

\section{Assessments}

Efficacy analyses were performed in the intent-to-treat (ITT) population (all patients who received $\geq 1$ sorafenib dose and had $\geq 1$ valid efficacy evaluation post-baseline). The primary endpoint was the ORR (complete or partial response) at 6 months in patients with $\geq 4$ months of therapy at the highest tolerated dose; this was analysed in the modified ITT (mITT) population (subgroup treated for $\geq 6$ months with $\geq 4$ months at their highest tolerated sorafenib dose). Secondary endpoints included PFS, time to progression (TTP), safety and tolerability, and pharmacokinetics. The safety population included all patients who received $\geq 1$ sorafenib dose and for whom post-baseline data were available. 
Tumour response and progression were assessed by central, independent, radiological review every 8 weeks using RECIST v1.0 [11]. Objective responses or stable disease were confirmed at the next scheduled scan. PFS was assessed from the start of study medication to the first radiological or clinical progression, or death. The TTP was measured from the start of study medication to the first radiological or clinical progression. AEs were assessed using the National Cancer Institute's Common Terminology Criteria for Adverse Events v3.0 [12]. Pharmacokinetic samples $(6 \mathrm{~mL})$ were collected on day 28 of the first completed cycle at each dose level at pre-dose and 2, 4, 6, 8, 10, and $12 \mathrm{~h}$ post-dose time points. Samples to measure plasma levels of sorafenib and its metabolites (M2, M4, and M5) were drawn on day 28 of the first cycle at each dose level.

\section{Determination of Sample Size}

A sample size of 80 patients was chosen in order to get a $95 \% \mathrm{CI} \pm 10 \%$ for a response rate of $\sim 30 \%$ (nQuery version 6.1 module POCO-1).

\section{Results}

\section{Patients}

The first patient was treated on 4 February 2008; the last patient visit was 13 January 2011; and the data collection limit was 6 August 2012. In all, 89 patients enrolled at 19 centres in France, UK, Germany, Italy, and Poland. Of these, 83 patients received sorafenib and were included in the safety population (Fig. S1). The ITT and mITT populations included 67 and 18 patients, respectively. Of the 49 ITT patients excluded from the mITT population, 32 (65.3\%) and 14 (28.6) discontinued sorafenib due to disease progression and toxicity, respectively (Table S7).

The ITT subgroups according to the sorafenib dose received for the longest duration showed broadly similar baseline demographics (Table 1). The mean time since diagnosis was 2.0, 0.7, and 2.3 years with 400, 600, and $800 \mathrm{mg}$ BID dose, respectively.

\section{Treatment Duration and Doses Received}

In the safety population, the median (range) treatment duration was 225 (7-1072) days, mean (SD) daily dose was 902 (364) $\mathrm{mg} / \mathrm{day}$, and the median (range) duration of follow-up was 252 (14-1 071) days. The maximum dose reached was $400 \mathrm{mg}$ BID in $31(37.3 \%)$ patients, $600 \mathrm{mg}$ BID in $12(14.5 \%)$ patients, and $800 \mathrm{mg}$ BID in $40(48.2 \%)$ patients. The dose [median (range) duration] received for the longest duration was $400 \mathrm{mg}$ BID in 40 (48.2\%) patients [29.5 (7-855) days]; $600 \mathrm{mg}$ BID in 13 (15.7\%) patients [164 (62-681) days]; $800 \mathrm{mg}$ BID in 20 (24.1\%) patients [177.5

Table 1 Baseline demographic and clinical characteristics.

\begin{tabular}{|c|c|c|c|c|c|}
\hline & \multirow{2}{*}{$\begin{array}{l}\text { Safety population } \\
\text { Overall }(n=83)\end{array}$} & \multicolumn{4}{|c|}{ III population } \\
\hline & & Overall $(n=67)$ & $\begin{array}{c}400 \mathrm{mg} \\
\mathrm{BID}^{*}(n=25)\end{array}$ & $\begin{array}{c}600 \mathrm{mg} \\
\mathrm{BID}^{*}(n=12)\end{array}$ & $\begin{array}{c}800 \mathrm{mg} \\
\mathrm{BID}^{*}(n=20)\end{array}$ \\
\hline Median (range) age, years & $61(33-80)$ & $62(33-80)$ & $64(44-80)$ & $59(33-78)$ & $57(39-72)$ \\
\hline Male, $n(\%)$ or $n / N$ & $54(65.1)$ & $44(65.7)$ & $15(60.0)$ & $9 / 12$ & $15(75.0)$ \\
\hline \multicolumn{6}{|l|}{ ECOG PS, $n(\%)$ or $n / N$} \\
\hline 0 & $49(59.0)$ & $40(59.7)$ & $14(56.0)$ & $7 / 12$ & $13(65.0)$ \\
\hline 1 & $34(41.0)$ & $27(40.3)$ & $11(44.0)$ & $5 / 12$ & $7(35.0)$ \\
\hline \multicolumn{6}{|l|}{ Disease stage, $n(\%)$ or $n / N$} \\
\hline III & $1(1.2)$ & $1(1.5)$ & 0 & 0 & 0 \\
\hline IV & $82(98.8)$ & $66(98.5)$ & $25(100.0)$ & $12 / 12$ & $20(100.0)$ \\
\hline \multicolumn{6}{|l|}{ Clinical/radiological status at entry, $n(\%)$ or $n / N$} \\
\hline Stable disease & $15(18.1)$ & $12(17.9)$ & $4(16.0)$ & $2 / 12$ & $2(10.0)$ \\
\hline Progressive disease & $68(81.9)$ & $55(82.1)$ & $21(84.0)$ & $10 / 12$ & $18(90.0)$ \\
\hline Mean (SD) time since initial diagnosis ${ }^{\dagger}$, years & $2.1(3.1)$ & $2.0(3.1)$ & $2.0(3.9)$ & $0.7(0.7)$ & $2.3(3.0)$ \\
\hline \multicolumn{6}{|l|}{ Number of metastatic lesions, $n(\%)$ or $n / N$} \\
\hline 1 & $14(16.9)$ & 0 & 0 & 0 & 0 \\
\hline$\geq 2$ & $69(83.1)$ & $67(100.0)$ & $25(100.0)$ & $12 / 12$ & $20(100.0)$ \\
\hline \multicolumn{6}{|l|}{ Metastatic sites, $n(\%)$ or $n / N$} \\
\hline Lung & $53(63.9)$ & $51(76.1)$ & $20(80.0)$ & $11 / 12$ & $14(70.0)$ \\
\hline Lymph nodes & $33(39.8)$ & $33(49.3)$ & $10(40.0)$ & $7 / 12$ & $11(55.0)$ \\
\hline Liver & $25(30.1)$ & $25(37.3)$ & $8(32.0)$ & $4 / 12$ & $9(45.0)$ \\
\hline Bone & $16(19.3)$ & $16(23.9)$ & $9(36.0)$ & $2 / 12$ & $3(15.0)$ \\
\hline \multicolumn{6}{|l|}{ Prior therapy for RCC, $n(\%)$ or $n / N$} \\
\hline Surgery & $83(100.0)$ & $67(100.0)$ & $25(100.0)$ & $12 / 12$ & $20(100.0)$ \\
\hline Radiotherapy & $12(14.5)$ & $10(14.9)$ & $4(16.0)$ & $3 / 12$ & $1(5.0)$ \\
\hline Systemic anticancer therapy ${ }^{\ddagger}$ & $3(3.6)$ & $2(3.0)$ & $1(4.0)$ & $0 / 12$ & 0 \\
\hline
\end{tabular}

${ }^{*}$ Dose taken for the longest duration while in the study; 10 patients treated at doses $<400 \mathrm{mg}$ BID are not included because of small sample sizes; ${ }^{\dagger}$ These data were unavailable for

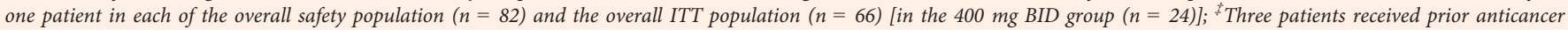
therapy with endocrine therapy $(n=2)$ and an immunostimulant $(n=1)$. These treatments were not considered protocol violations. 
(56-956) days]; $400 \mathrm{mg}$ daily in seven (8.4\%) patients [434 (122-764) days]; and $400 \mathrm{mg}$ every other day in three (3.6\%) patients [332 (136-675) days].

\section{Efficacy}

In the mITT population, all patients had partial response ( $n$ $=8 / 18)$ or stable disease $(n=10 / 18)$ (Table $2 \mathrm{~A})$. The primary efficacy endpoint, the ORR, was $44.4 \%$ (95\% CI 21.5-69.2). In the ITT population, the ORR was $17.9 \%(n=12 / 67)(95 \% \mathrm{CI}$ 9.629 .2 ) (Table $2 B$ ). Tumour shrinkage occurred in $18 / 25$ (72.0\%), (9/12) $75.0 \%$, and $17 / 20(85.0 \%)$ of patients in the 400, 600, and $800 \mathrm{mg}$ BID groups, respectively (Fig. 1).

In the ITT population, the overall median PFS was 7.4 months (95\% CI 6.0 11.7) (Table 2B and Fig. 2 [3]); $62.3 \%$ and $33.4 \%$ of patients were progression free at 6 and 12 months, respectively. The median (95\% CI) PFS was 3.7 (1.8-9.7), $7.4(6.3-12.0)$, and 8.5 (5.5-14.9) months for the 400,600 , and $800 \mathrm{mg}$ BID groups, respectively (ITT). The TTP results were identical to those for PFS, because no deaths occurred before disease progression was observed.

\section{Safety}

All 83 patients reported at least one treatment-emergent $\mathrm{AE}$ (TEAE). The most common TEAEs of any grade were handfoot skin reaction (HFSR; 66.3\%), diarrhoea (63.9\%), rash/ desquamation (56.6\%), fatigue (54.2\%), and hypertension (48.2\%) (Table 3). One patient (1.2\%) had Grade 2 proteinuria. Most patients $(90.4 \% ; n=75)$ had at least one $\geq$ Grade 3 event. The most common Grade 3 events were HFSR (25.3\%), fatigue (15.7\%), hypophosphataemia (15.7\%), and rash/desquamation (13.3\%) (Table 3). Two patients had Grade 3 renal failure. Apart from hyponatraemia and elevated lipase [both two patients (2.4\%)], Grade 4 events occurred in individual patients only (Table 3). Table 4 summarises the TEAEs by dose at first occurrence. Most patients (91.6\%) had their first AE at $400 \mathrm{mg}$ BID.

Serious TEAEs were reported in 44 (53.0\%) patients and most were single occurrences. The most common serious TEAEs, each occurring in three (3.6\%) patients, were fatigue, rash/ desquamation, gastrointestinal (other), hyponatraemia, and intraoperative injury.

Dose interruptions, reductions, and withdrawals due to AEs occurred in 69 (83.1\%), 50 (60.2\%), and $36(43.4 \%)$ patients, respectively. Dose interruptions or withdrawals occurred most frequently in patients receiving $400 \mathrm{mg}$ BID vs other doses.

One death was reported, due to cardiopulmonary failure, which was not considered to be related to sorafenib. Another death was reported $>30$ days after the last study drug dose due to cardiopulmonary failure caused by progressive RCC.

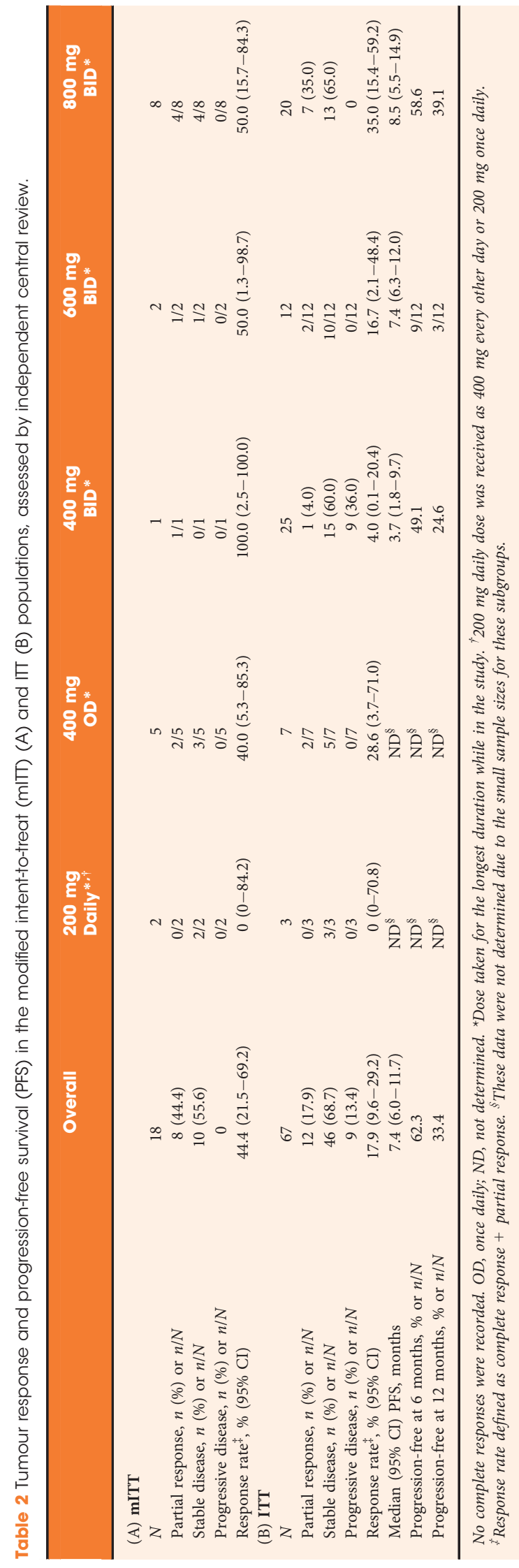


Fig. 1 Maximum tumour shrinkage in individual patients (\% change from baseline in target lesions by independent assessment) according to the dose received for the longest duration in the study [intent-to-treat (ITT) population]. Dotted line represents the threshold for response using Response Evaluation Criteria in Solid Tumors (RECIST) v1.0. Maximum tumour shrinkage for patients in the IT population who received doses of 400,600 , or $800 \mathrm{mg} /$ day twice daily (BID) for the longest duration while in the study were included as long as the independent central reviewer could establish a best response.

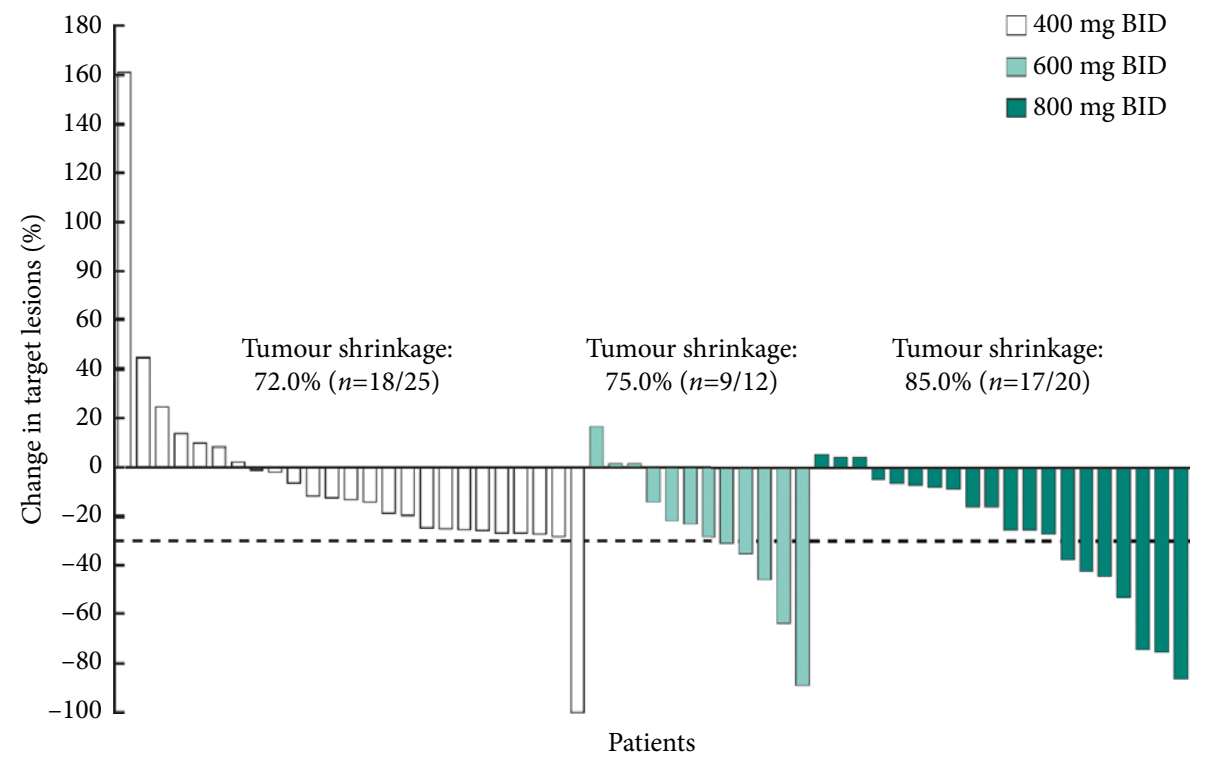

In both cases, the sorafenib dose received for the longest duration was $400 \mathrm{mg}$ BID.

\section{Pharmacokinetics}

No increase in exposure [area under the curve (AUC) or maximum plasma concentration $\left.\left(C_{\max }\right)\right]$ for sorafenib or its metabolites (M2, M4, and M5) was observed with increase in dose, indicating a lack of dose proportionality

(Table S8).

\section{Discussion}

In the present open-label dose-escalation study, sorafenib showed apparent clinical benefit in the ORR and PFS in treatment-naive patients with $\mathrm{mRCC}$ in the mITT population. The ORR for the mITT population (eight of 18 patients, $44.4 \%$ ) compared favourably to that in other first-line sorafenib trials $[3,8,13-17]$, and was similar to that in the Phase 2 dose-escalation study of Amato et al. [9] of $47.7 \%$. These observations suggest that mITT patients may have gained additional benefit from sorafenib dose escalation. However, these results should be interpreted cautiously due to small patient numbers and the fact that, by definition, the mITT population had tolerated sorafenib relatively well ( $\geq 4$ months at the maximum tolerated dose) and had slowgrowing tumours (stayed in the study for $\geq 6$ months). The mITT population may only represent a small proportion of patients with mRCC.
Fig. 2 Kaplan-Meier graph showing progression-free survival (PFS) by independent central assessment according to the dose received for the longest duration in the study [intent-to-treat (ITT) population]. The PFS curve from the Phase 3 trial of sorafenib for treatment of metastatic renal cell carcinoma (mRCC) (TARGET) is shown for comparison [3].

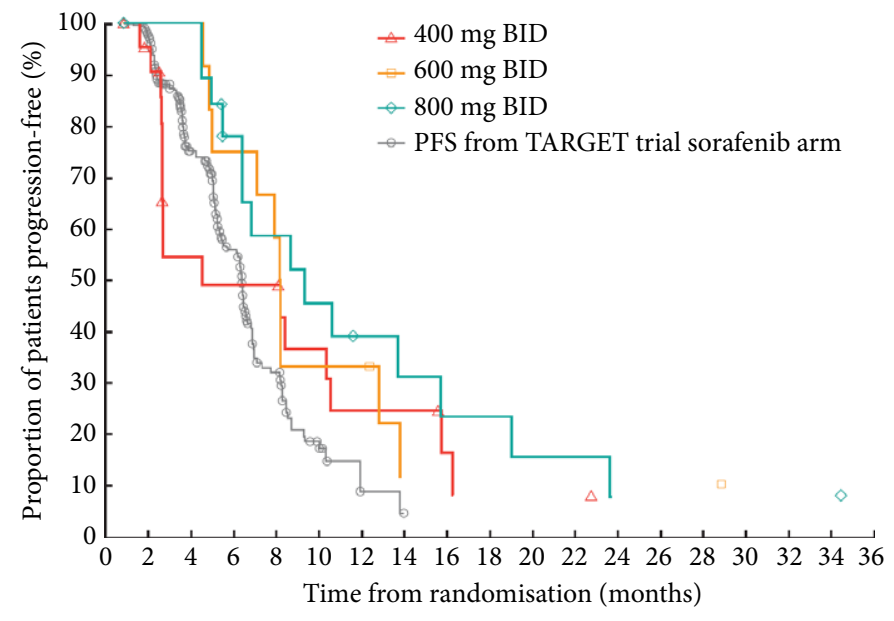

In the ITT population, the median PFS (7.4 months) and ORR (17.9\%) fell within the ranges reported in Phase $2 / 3$ studies of first-line standard-dose sorafenib in mRCC (median PFS 5.5-9.1 months; ORR 5.2-30.0\%) [3,8,13-17]. Therefore, the results of the present study do not support an improved benefit/risk ratio with sorafenib dose escalation up to $800 \mathrm{mg}$ BID in mRCC compared to historical data with 
Table 3 Incidence of TEAEs by worst grade, occurring in $>10 \%$ patients at any grade, $>5 \%$ patients at Grade 3 , or $>2 \%$ patients at Grade 4 (safety population, $N=83$ ).

\begin{tabular}{|c|c|c|c|}
\hline \multirow[t]{2}{*}{ Adverse event } & \multicolumn{3}{|c|}{$n(\%)$} \\
\hline & Any grade & Grade 3 & Grade 4 \\
\hline Any event & $83(100.0)$ & $61(73.5)$ & $13(15.7)$ \\
\hline HFSR & $55(66.3)$ & $21(25.3)$ & 0 \\
\hline Diarrhoea & $53(63.9)$ & $10(12.0)$ & $1(1.2)$ \\
\hline Rash/desquamation & $47(56.6)$ & $11(13.3)$ & 0 \\
\hline Fatigue & $45(54.2)$ & $13(15.7)$ & $1(1.2)$ \\
\hline Hypertension & $40(48.2)$ & $5(6.0)$ & 0 \\
\hline Alopecia & $36(43.4)$ & 0 & 0 \\
\hline Mucositis (functional/symptomatic), oral cavity & $27(32.5)$ & 0 & 0 \\
\hline Dry skin & $23(27.7)$ & $1(1.2)$ & 0 \\
\hline Nausea & $22(26.5)$ & 0 & 0 \\
\hline Anorexia & $21(25.3)$ & $1(1.2)$ & 0 \\
\hline Hypophosphataemia & $17(20.5)$ & $13(15.7)$ & $1(1.2)$ \\
\hline Vomiting & $16(19.3)$ & $1(1.2)$ & 0 \\
\hline Pruritus & $15(18.1)$ & $1(1.2)$ & 0 \\
\hline Fever & $14(16.9)$ & 0 & 0 \\
\hline Weight loss & $14(16.9)$ & $1(1.2)$ & 0 \\
\hline Dyspnoea & $13(15.7)$ & $1(1.2)$ & 0 \\
\hline Haemoglobin & $12(14.5)$ & $1(1.2)$ & 0 \\
\hline Hypothyroidism & $12(14.5)$ & 0 & 0 \\
\hline Neuropathy: sensory & $12(14.5)$ & 0 & 0 \\
\hline Pain, abdomen (not otherwise specified) & $11(13.3)$ & $1(1.2)$ & 0 \\
\hline Taste alteration & $11(13.3)$ & 0 & 0 \\
\hline Voice changes & $11(13.3)$ & 0 & 0 \\
\hline Lipase & $10(12.0)$ & $10(12.0)$ & $2(2.4)$ \\
\hline Pain, back & $10(12.0)$ & $3(3.6)$ & 0 \\
\hline Constipation & $9(10.8)$ & 0 & 0 \\
\hline Alanine aminotransferase & $7(8.4)$ & $5(6.0)$ & 0 \\
\hline Hyponatraemia & $5(6.0)$ & $3(3.6)$ & $2(2.4)$ \\
\hline
\end{tabular}

Table 4 Incidence of TEAEs (any grade, occurring in $>10 \%$ of patients in any category) by dose at first occurrence (safety population).

\begin{tabular}{|c|c|c|c|c|c|}
\hline \multirow[t]{2}{*}{$\mathbf{A E}$} & \multicolumn{5}{|c|}{$n / N$ or $n(\%)$} \\
\hline & $\begin{array}{l}200 \mathrm{mg} \\
\text { Daily* }(n=10)\end{array}$ & $\begin{array}{l}400 \mathrm{mg} \\
\text { OD }(n=38)\end{array}$ & $\begin{array}{l}400 \mathrm{mg} \\
\text { BID }(n=83)\end{array}$ & $\begin{array}{l}600 \mathrm{mg} \\
\operatorname{BID}(n=52)\end{array}$ & $\begin{array}{l}800 \mathrm{mg} \\
\text { BID }(n=40)\end{array}$ \\
\hline Any event & $1 / 10$ & $5(13.2)$ & $76(91.6)$ & 0 & $1(2.5)$ \\
\hline HFSR & $0 / 10$ & $3(7.9)$ & $43(51.8)$ & $5(9.6)$ & $4(10.0)$ \\
\hline Rash/desquamation & $1 / 10$ & $1(2.6)$ & $38(45.8)$ & $5(9.6)$ & $2(5.0)$ \\
\hline Fatigue & $2 / 10$ & $2(5.3)$ & $29(34.9)$ & $4(7.7)$ & $5(12.5)$ \\
\hline Hypertension & $2 / 10$ & $4(10.5)$ & $29(34.9)$ & $2(3.8)$ & $2(5.0)$ \\
\hline Diarrhoea & $1 / 10$ & $3(7.9)$ & $21(25.3)$ & $19(36.5)$ & $9(22.5)$ \\
\hline Oral mucositis (functional/symptomatic) & $1 / 10$ & $2(5.3)$ & $19(22.9)$ & $4(7.7)$ & $1(2.5)$ \\
\hline Alopecia & $1 / 10$ & $5(13.2)$ & $16(19.3)$ & $9(17.3)$ & $5(12.5)$ \\
\hline Dry skin & $1 / 10$ & $1(2.6)$ & $13(15.7)$ & $3(5.8)$ & $5(12.5)$ \\
\hline Hypophosphataemia & $0 / 10$ & $1(2.6)$ & $13(15.7)$ & $3(5.8)$ & 0 \\
\hline Nausea & $2 / 10$ & $2(5.3)$ & $10(12.0)$ & $3(5.8)$ & $5(12.5)$ \\
\hline Pruritus & $0 / 10$ & $2(5.3)$ & $10(12.0)$ & $2(3.8)$ & 0 \\
\hline Vomiting & $0 / 10$ & $1(2.6)$ & $8(9.6)$ & $2(3.8)$ & $5(12.5)$ \\
\hline Anorexia & $1 / 10$ & 0 & $6(7.2)$ & $8(15.4)$ & $6(15.0)$ \\
\hline Weight loss & $0 / 10$ & 0 & $5(6.0)$ & $2(3.8)$ & $7(17.5)$ \\
\hline Hypocalcaemia & $0 / 10$ & 0 & $1(1.2)$ & 0 & $5(12.5)$ \\
\hline
\end{tabular}

Data are ordered in decreasing incidence seen in the largest subgroup (400 mg BID). OD, once daily. ${ }_{2} 00 \mathrm{mg}$ daily dose was received as $400 \mathrm{mg}$ every other day or $200 \mathrm{mg}$ OD.

sorafenib $400 \mathrm{mg}$ BID. However, comparison of ORR and PFS in different dose groups showed that patients who tolerated higher doses of sorafenib ( $>400 \mathrm{mg}$ BID) appeared to have enhanced clinical benefit compared with those receiving doses of $\leq 400 \mathrm{mg}$ BID. However, a meaningful comparison between the dosage groups is limited because the higher-dose groups were enriched with patients who tolerated sorafenib better and whose disease progressed later.

Outcomes in the ITT population appeared inferior to those reported by Amato et al. [9], who followed a similar 
dose-escalation protocol [9]. This may reflect the fact that sorafenib therapy and dose escalation were less well tolerated in the present study. Of note, the numbers of Grade 3/4 AEs in the Amato et al. study were much lower, allowing a greater proportion of patients to reach and potentially benefit from the $800 \mathrm{mg}$ BID dose. Patients in the Texan Amato et al. [9] study could have been more homogeneous, and different in anthropometric characteristics, compared with our international ITT population.

Dosage groups for pharmacokinetics were not the same as for efficacy (dose received for the longest duration) or safety (dose at first occurrence). Rather, blood for pharmacokinetic analyses was collected on day 28 of the first cycle completed at each dose level. No apparent increase in sorafenib exposure was seen at higher doses. However, patients were not randomised into dose groups, there is large inter-patient variation in sorafenib exposure at the same dose, and incidence of Grade 3/4 AEs has been associated with higher exposure $[18,19]$. Patients with low sorafenib exposure may therefore have been over-represented in the high-dose groups, being less prone to severe AEs that precluded dose escalation. Further confounding interpretation of pharmacokinetic data, samples from patients receiving higher doses were taken at later time points than lower-dose samples, and sorafenib exposure declines over time $[19,20]$.

No new or unexpected toxicities arose in our present study. Most TEAEs first occurred with the starting dose of sorafenib, $400 \mathrm{mg}$ BID, which is consistent with previous analyses showing that AEs with sorafenib tend to first occur early in treatment $[21,22]$. Gastrointestinal disorders were the exception, most often starting with 600 or 800 mg BID. These findings should be interpreted cautiously given that patients were not randomised to dose groups. However, these observations are consistent with data from a sorafenib doseescalation study in metastatic melanoma, where HFSR and hypertension correlated with exposure, whereas diarrhoea and anorexia correlated with dose level [23]. The small proportion of patients who could sustain the highest dose level and the need for frequent dose reductions and interruptions to manage AEs reflects the difficulties of generally implementing a dose-escalation schedule in this patient population. However, there may be value in exploring alternative protocols for sorafenib dose escalation, e.g., escalation to restore antitumour activity in patients whose disease progressed with reduced exposure, or regular monitoring of plasma concentrations and dose adjustment to maintain exposure over time $[20,24]$.

The present study assessed the use of scheduled intra-patient dose escalation to enhance response rates with sorafenib in patients with mRCC. We conclude that escalating the sorafenib dose from the standard $400 \mathrm{mg}$ BID may have benefited individual patients able to tolerate this approach.
However, the present study does not support this type of scheduled dose escalation for all patients with treatment-naïve mRCC. Alternative protocols for sorafenib dose escalation could be explored.

\section{Acknowledgements}

We thank all the patients, caregivers, and investigators who participated in this study. We also thank Jenny Handford and Jerry King from 7.4 Limited, who provided medical writing support funded by Bayer HealthCare Pharmaceuticals. The sponsor played a role in design and conduct of the study, collection, management, analysis, and interpretation of the data, and review and approval of the manuscript, and funded medical writing support for the preparation of this article.

\section{Author Contributions}

M.E. Gore and M. Kuczyk contributed to the study concept and design. A. Bearz, T. Demkow, M.E. Gore, R.J. Jones, C. Porta, and A. Ravaud acquired the data. M.E. Gore, M. Kuczyk, C. Porta, J. Shapiro, and U.P. Strauss analysed and interpreted the data. M.E. Gore, M. Kuczyk, and U.P. Strauss drafted the manuscript. T. Demkow, M.E. Gore, R.J. Jones, M. Kuczyk, C. Porta, A. Ravaud, J. Shapiro, and U.P. Strauss critically reviewed the manuscript for important intellectual content. J. Shapiro did the statistical analysis. R.J. Jones and M. Kuczyk provided administrative, technical, or material support.

\section{Conflicts of Interest}

M.E. Gore is funded by the NIHR Biomedical Research Centre at the Royal Marsden NHS Foundation Trust and Institute of Cancer Research, London; he received honoraria from Bayer for advisory boards and contributions to speaker bureaux during the course of this trial. R.J. Jones has received honoraria from Bayer, Pfizer, Novartis, Roche and Bristol Myers for advisory boards. His institution has received research funding from Bayer, Pfizer, Novartis, GSK and Roche. A. Ravaud received honoraria from Bayer for advisory boards during the course of this trial, speaker honoraria from Pfizer, Novartis, Roche, Bristol Myers and GSK and institutional grants from Pfizer and Novartis. M. Kuczyk has acted as a consultant and/or speaker for Bayer, Pfizer, BMS, and Novartis. J. Shapiro is an employee of Bayer and owns Bayer stock. U.P. Strauss is an employee of Bayer. C. Porta has acted as a consultant and/or speaker for Bayer, Pfizer, Novartis, BMS, GSK, Exelixis, Genentech-Roche, Astellas, and Pierre-Fabre; he has also received research funding (for his Institution) from Pfizer and Novartis. T. Demkow and A. Bearz have nothing to declare.

\section{References}

1 Brose MS, Nutting CM, Jarzab B et al. Sorafenib in radioactive iodinerefractory, locally advanced or metastatic differentiated thyroid cancer: a randomised, double-blind, phase 3 trial. Lancet 2014; 384: 319-28 
2 Llovet JM, Ricci S, Mazzaferro V et al. Sorafenib in advanced hepatocellular carcinoma. N Engl J Med 2008; 359: 378-90

3 Escudier B, Eisen T, Stadler WM et al. Sorafenib in advanced clear-cell renal-cell carcinoma. N Engl J Med 2007; 356: 125-34

4 Escudier B, Eisen T, Stadler WM et al. Sorafenib for treatment of renal cell carcinoma: final efficacy and safety results of the phase III treatment approaches in renal cancer global evaluation trial. J Clin Oncol 2009; 27: 3312-8

5 FDA. Nexavar US Prescribing Information. Available at: http://www.acce ssdata.fda.gov/drugsatfda_docs/label/2013/021923s016lbl.pdf. Accessed June 2015

6 EMA. Nexavar Summary of Product Characteristics. Available at: http:// www.ema.europa.eu/humandocs/Humans/EPAR/nexavar/nexavar.htm. Accessed June 2013

7 Semrad TJ, Gandara DR, Lara PN Jr. Enhancing the clinical activity of sorafenib through dose escalation: rationale and current experience. Ther Adv Med Oncol 2011; 3: 95-100

8 Escudier B, Szczylik C, Hutson TE et al. Randomized phase II trial of first-line treatment with sorafenib versus interferon Alfa-2a in patients with metastatic renal cell carcinoma. J Clin Oncol 2009; 27: 1280-9

9 Amato R, Zhai J, Willis J, Saxena S, Defoe M. A Phase II trial of intrapatient dose-escalated sorafenib in patients with metastatic renal cell carcinoma. Clin Genitourin Cancer 2012; 10: 153-8

10 Motzer RJ, Mazumdar M, Bacik J, Berg W, Amsterdam A, Ferrara J. Survival and prognostic stratification of 670 patients with advanced renal cell carcinoma. J Clin Oncol 1999; 17: 2530-40

11 Therasse P, Arbuck SG, Eisenhauer EA et al. New guidelines to evaluate the response to treatment in solid tumors. European Organization for Research and Treatment of Cancer, National Cancer Institute of the United States, National Cancer Institute of Canada. J Natl Cancer Inst 2000; 92: 205-16

12 National Cancer Institute. Common Terminology Criteria for Adverse Events (CTCAE) v3.0. Available at: http://ctep.cancer.gov/protocolDeve lopment/electronic_applications/ctc.htm\#ctc_archive. Accessed June 2013

13 Jonasch E, Corn P, Pagliaro LC et al. Upfront, randomized, phase 2 trial of sorafenib versus sorafenib and low-dose interferon Alfa in patients with advanced renal cell carcinoma: clinical and biomarker analysis. Cancer 2010; 116: 57-65

14 Rini BI, Szczylik C, Tannir NM et al. AMG 386 in combination with sorafenib in patients with metastatic clear cell carcinoma of the kidney: a randomized, double-blind, placebo-controlled, phase 2 study. Cancer 2012; 118: 6152-61

15 Procopio G, Verzoni E, Bracarda S et al. Sorafenib with interleukin-2 vs sorafenib alone in metastatic renal cell carcinoma: the ROSORC trial. $\mathrm{Br} J$ Cancer 2011; 104: 1256-61

16 Motzer RJ, Nosov D, Eisen T et al. Tivozanib versus sorafenib as initial targeted therapy for patients with metastatic renal cell carcinoma: results from a phase III trial. J Clin Oncol 2013; 31: 3791-9

17 Hutson TE, Lesovoy V, Al-Shukri S et al. Axitinib versus sorafenib as first-line therapy in patients with metastatic renal-cell carcinoma: a randomised open-label phase 3 trial. Lancet Oncol 2013; 14: 128794

18 Shimada M, Okawa H, Kondo Y et al. Monitoring serum levels of sorafenib and its $\mathrm{N}$-oxide is essential for long-term sorafenib treatment of patients with hepatocellular carcinoma. Tohoku J Exp Med 2015; 237: 173-82

19 Boudou-Rouquette P, Ropert S, Mir O et al. Variability of sorafenib toxicity and exposure over time: a pharmacokinetic/pharmacodynamic analysis. Oncologist 2012; 17: 1204-12
20 Arrondeau J, Mir O, Boudou-Rouquette P et al. Sorafenib exposure decreases over time in patients with hepatocellular carcinoma. Invest New Drugs 2012; 30: 2046-9

21 Worden F, Fassnacht M, Shi Y et al. Safety and tolerability of sorafenib in patients with radioiodine-refractory thyroid cancer. Endocr Relat Cancer 2015; 22: 877-87

22 Hutson TE, Bellmunt J, Porta C et al. Long-term safety of sorafenib in advanced renal cell carcinoma: follow-up of patients from phase III TARGET. Eur J Cancer 2010; 46: 2432-40

23 Pecuchet N, Lebbe C, Mir O et al. Sorafenib in advanced melanoma: a critical role for pharmacokinetics? Br J Cancer 2012; 107: 455-61

24 Bellesoeur A, Carton E, Mir O et al. Critical role of sorafenib exposure over time for its antitumor activity in thyroid cancer. Invest New Drugs 2014; 32: 569-72

Correspondence: Martin E. Gore, The Royal Marsden NHS Foundation Trust, Department of Medical Oncology, Mulberry House, Fulham Road, London SW3 6JJ, UK.

e-mail: martin.gore@rmh.nhs.uk

Abbreviations: AUC, area under the curve; BID, twice daily; $C_{\text {max }}$, maximum plasma concentration; ECOG PS, Eastern Cooperative Oncology Group Performance status; G-CSF, granulocyte-colony stimulating factor; HFSR, hand-foot skin reaction; (m)ITT, (modified) intent-to-treat; mRCC, metastatic RCC; ORR, objective response rate; PFS, progression-free survival; RECIST, Response Evaluation Criteria in Solid Tumors; (TE)AE, (treatment-emergent) adverse event; TTP, time to progression.

\section{Supporting Information}

Additional Supporting Information may be found in the online version of this article:

Figure S1. Patient flow.

Table S1. Name of approving ethics committees.

Table S2. Predefined dose modification levels for treatment with sorafenib.

Table S3. Criteria for sorafenib dose delay and dose modification due to skin toxicities.

Table S4. Management of treatment-emergent hypertension. Table S5. Criteria for dose delay and dose modification due to haematological adverse events.

Table S6. Criteria for dose delay and dose modification due to non-haematological adverse events (except skin toxicity) ${ }^{\mathrm{a}}$.

Table S7. Reasons for permanent discontinuation of sorafenib for patients in the intent-to-treat population who did not qualify for the modified intent-to-treat population by last dose received.

Table S8. Pharmacokinetic parameters for sorafenib and its metabolites (M2, M4, and M5) after $28 \mathrm{~d}$ of sorafenib 400, 600 , or $800 \mathrm{mg}$ BID (all patients valid for pharmacokinetics). 